21

\title{
Терагерцовое излучение фотопроводящих антенн на основе сверхрешеточных структур \{LT-GaAs/GaAs:Si\}
}

\author{
(C) А.Н. Клочков ${ }^{1}$, Е.А. Климов ${ }^{1}$, П.М. Солянкин ${ }^{2}$, М.Р. Конникова ${ }^{2}$, И.С. Васильевский ${ }^{4}$, \\ А.Н. Виниченко ${ }^{4}$, А.П. Шкуринов ${ }^{2,3}$, Г.Б. Галиев ${ }^{1}$ \\ ${ }^{1}$ Институт сверхвысокочастотной полупроводниковой электроники имени В.Г. Мокерова РАН, \\ 117105 Москва, Россия \\ ${ }^{2}$ Институт проблем лазерных и информационных технологий РАН - филиал Федерального государственного \\ учреждения - Федеральный научно-исследовательский центр „Кристаллография и фротоника“ РАН, \\ 140700 Шатура, Московская обл., Россия \\ ${ }^{3}$ Физический фракультет и международный лазерный центр, \\ Московский государственный университет им. М.В. Ломоносова, \\ 119992 Москва, Россия \\ ${ }^{4}$ Национальный исследовательский ядерный университет „МИФИ“, \\ 115409 Москва, Россия \\ e-mail: klochkov_alexey@mail.ru
}

Поступила в редакцию 16.12.2019 г.

В окончательной редакции 13.01.2020 г.

Принята к публикации 28.02.2020 г.

Предложен материал в виде многослойной структуры на основе низкотемпературного LT-GaAs, выращенного на подложках с ориентацией (111)A, для изготовления терагерцовых (ТГц) фотопроводящих антенн. Структуры содержат активные слои из LT-GaAs вместе с легирующими акцепторными слоями на основе GaAs:Предложен материал в виде многослойной структуры на основе низкотемпературного LT-GaAs, выращенного на подложках с ориентацией (111)A, для изготовления терагерцовых (ТГц) фотопроводящих антенн. Структуры содержат активные слои из LT-GaAs вместе с легирующими акцепторными слоями на основе GaAs: Si. При мощности оптической накачки $19 \mathrm{~mW}$ и напряжении смещения $30 \mathrm{~V}$ фотопроводящая антенна на оптимизированной структуре $\{\mathrm{LT}-\mathrm{GaAs} / \mathrm{GaAs}: \mathrm{Si}\}$ (111)A испускала ТГц импульсы со средней мощностью $2.3 \mu \mathrm{W}$ при частоте следования импульсов $80 \mathrm{MHz}$, эффективность преобразования составила $1.2 \cdot 10^{-4}$. Показано, что зависимость интегральной мощности ТГц импульсов антенны на основе \{LT$\mathrm{GaAs} / \mathrm{GaAs}: \mathrm{Si}\}$ (111)A структуры от приложенного напряжения является суперлинейной, а от мощности оптической накачки имеет вид кривой насыщения. Показана возможность практического применения полученных антенн для задач терагерцовой спектроскопии биологических растворов.

Ключевые слова: импульсная терагерцовая спектроскопия, фотопроводящая антенна, низкотемпературный GaAs.

DOI: $10.21883 /$ OS.2020.07.49574.17-20

\section{Введение}

В последнее время появилась актуальность решения задачи повышения мощности источников импульсного широкополосного терагерцового (ТГц) излучения, испускаемого при возбуждении фемтосекундными оптическими импульсами [1]. Наиболее широко используемыми источниками и детекторами импульсного ТГц излучения являются фотопроводящие антенны (ФПА) [2]. При облучении ФПА фемтосекундными лазерными импульсами в фотопроводящем материале рождаются неравновесные носители заряда. Под действием приложенного к ФПА электрического поля формируется переменный ток, который из-за малого времени жизни неравновесных носителей при затухании возбуждает колебания электромагнитного поля в микроволновом и терагерцовом диапазонах частот. Энергия и спектр ТГц импульсов определяется характеристиками оптических импульсов накачки, параметрами фотопроводящего материала и геометрией металлизации ФПА. В последнее время эффективность оптико-терагерцового преобразования энергии в ФПАисточниках и динамический диапазон ФПА-детекторов удалось существенно повысить путем использования металлических наноструктур, таких как оптические наноантенны $[3,4]$ и плазмонные наноэлектроды [5]. Рекордная эффективность преобразования 7.5\% была получена в плазмонных антеннах в работе [6].

Классическим фотопроводящим материалом для ФПА, работающих с Ti:Sa лазерами с длиной волны $800 \mathrm{~nm}$, является низкотемпературный (LT - low temperature) GaAs [7]. Он обладает ультракоротким временем захвата фотовозбужденных носителей заряда, большим темновым удельным сопротивлением и хорошей подвижностью электронов. Пониженная температура роста приводит к встраиванию избыточного As в решетку LT-GaAs и формированию ассоциированных c As дефектов [8]. При сохранении монокристаллической структуры пленок LT-GaAs точечные дефекты 
обеспечивают субпикосекундное время жизни носителей заряда [9]. In-situ отжиг пленок LT-GaAs позволяет оптимизировать такие характеристики фотопроводящего материала как темновое сопротивление и время релаксации для использования в ФПА $[10,11]$. Легирование примесными атомами в процессе эпитаксиального роста может привести к образованию в LT-GaAs новых „примесных“ точечных дефектов, примесных комплексов, изменяющих особенности динамики неравновесных носителей заряда при фотовозбуждении. Например, легирование акцепторными атомами Ве или С может существенно уменьшить время захвата электронов $[12,13]$. Считается, что акцепторы изменяют зарядовое состояние антиструктурных дефектов $\mathrm{AsGa}$, являющихся основными центрами безызлучательной рекомбинации в LT-GaAs [14].

Недавно для изготовления ФПА был предложен [15] материал на основе низкотемпературного (LT-) GaAs, содержащий сверхрешетку $\{\mathrm{LT}-\mathrm{GaAs} / \mathrm{GaAs}: \mathrm{Si}\}$, где слои LT-GaAs выращиваются при низких температурах $T_{G} \leq 300^{\circ} \mathrm{C}$, а легированные атомами кремния слои GaAs:Si - при более высоких. Данные структуры выращивались на подложках GaAs с ориентацией поверхности (111)A, которая при подборе условий эпитаксиального роста слоев $\mathrm{GaAs}: \mathrm{Si}$ обеспечивала акцепторное легирование. Использование подложек с ориентацией (111)А в ряде случаев приводило к увеличению амплитуды ТГц импульсов от пленок или антенн на основе низкотемпературных материалов $[16,17]$. В данной работе исследуются характеристики ФПА на основе структур $\{\mathrm{LT}-\mathrm{GaAs} / \mathrm{GaAs}: \mathrm{Si}\}$, полученных при различных эпитаксиальных условиях, а также демонстрируется возможность практического применения полученных антенн для задач терагерцовой спектроскопии водных белковых растворов на примере бычьего сывороточного альбумина.

\section{Образцы и методы исследования}

Образцы были выращены методом молекулярнолучевой эпитаксии (МЛЭ) на полуизолирующих подложках GaAs. Схематическое изображение поперечного сечения образцов представлено на рис. 1, $a$. После буферного слоя $i$-GaAs толщиной $200 \mathrm{~nm}$ выращивалась многослойная структура, состоящая из чередующихся слоев нелегированного LT-GaAs толщиной $230 \mathrm{~nm}$, синтезированных в низкотемпературном режиме, и GaAs, легированного $\mathrm{Si}(20 \mathrm{~nm})$. По существу, полученные структуры являются сверхрешетками легирования \{LT$\mathrm{GaAs} / \mathrm{GaAs}: \mathrm{Si}\}$. Особенности структуры образцов, исследованные методом просвечивающей электронной микроскопии, приведены в работе [18]. Слои LT-GaAs выращивались при $240^{\circ} \mathrm{C}$, а более высокотемпературные слои GaAs: $\mathrm{Si}$ - при $480^{\circ} \mathrm{C}[19]$.

Было выращено два типа структур \{LT-GaAs/GaAs : Si $\}$. Образцы ФПА № 7 и № 26 были получены при постоянном значении $\gamma=25$ соотношения парциальных

\begin{tabular}{|lc|}
\hline \multicolumn{1}{c}{$a$} \\
\hline GaAs:Si & $20 \mathrm{~nm}$ \\
\hline LT-GaAs & $230 \mathrm{~nm}$ \\
\hline GaAs:Si & $20 \mathrm{~nm}$ \\
\hline LT-GaAs & $230 \mathrm{~nm}$ \\
\hline GaAs:Si & $20 \mathrm{~nm}$ \\
\hline LT-GaAs & $230 \mathrm{~nm}$ \\
\hline GaAs:Si & $20 \mathrm{~nm}$ \\
\hline LT-GaAs & $230 \mathrm{~nm}$ \\
\hline$i$-GaAs buffer & $200 \mathrm{~nm}$ \\
\hline Substrate GaAs $(100)$ or $(111) \mathrm{A}$ \\
\hline
\end{tabular}
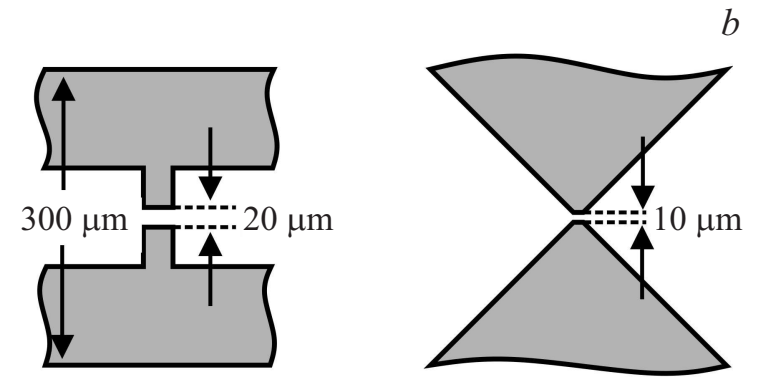

Рис. 1. Схема образцов: $a$ - поперечное сечение эпитаксиальных структур, $b$ - геометрия электродов дипольной (слева) и галстук-бабочка (справа) ФПА в окрестности возбуждаемого светом фотопроводящего зазора.

давлений молекул $\mathrm{As}_{4}$ и атомов Ga. Значение $\gamma=25$ было выбрано оптимальным для удовлетворения одновременно двух условий: получения проводимости $p$-типа в слоях GaAs:Si на подложках $\mathrm{GaAs}(111) \mathrm{A}$ и внедрения избыточного мышьяка при росте слоев LT-GaAs. В одном процессе образцы № 7 и № 26 выращивались на двух типах подложек GaAs (100) и (111)A. Сразу после роста структуры образец № 26 был подвергнут in situ отжигу при температуре $560^{\circ} \mathrm{C}$ в течение $30 \mathrm{~min}$ в потоке $\mathrm{As}_{4}$. Вторая разновидность структуры (образец № 443) выращивалась только на подложке (111)А. Образец № 443 отличался тем, что слои LTGaAs формировались при увеличенном значении $\gamma=60$, а слои GaAs: $\mathrm{Si}$ - при уменьшенном значении $\gamma=15$. Уменьшение $\gamma$ до 15 более надежно обеспечивает $p$-тип проводимости в слоях GaAs:Si (111)A. Увеличение $\gamma$ c 25 до 60 при росте слоев LT-GaAs обеспечивало увеличенную концентрацию точечных дефектов. Сразу после роста образец № 443 раскалывался на две части, одна из которых затем отжигалась в установке МЛЭ при условиях, аналогичных образцу № 26.

На поверхности структур методом фотолитографии были изготовлены планарные фотопроводящие антенны с дипольной геометрией либо геометрией в форме галстук-бабочка. Схема антенн в окрестности фотопроводящего зазора приведена на рис. $1, b$. Металлическая 

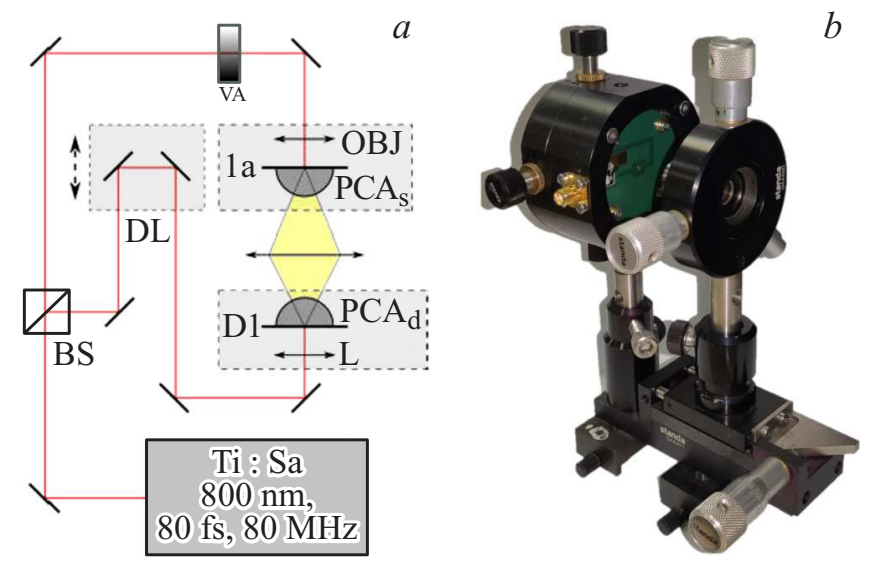

Рис. 2. Упрощенная схема установки для измерения ТГц сигнала $(a)$. BS - делительный куб, DL - линия задержки, VA - аттенюатор, OBJ - микрообъектив, L - линза, LTHz полипропиленовая ТГц линза, $\mathrm{PCA}_{\mathrm{s}}$ и $\mathrm{PCA}_{\mathrm{d}}-$ излучающая и детектирующая ФПА. Применялось два детектора: на основе ФПА (D1: L + PCAd) и на базе ячейки Голея (заменяет блок D1 на рисунке). Фотография юстируемого держателя ФПА (OBJ+PCAs) $(b)$.

композиция из $\mathrm{Ni} / \mathrm{Ge} / \mathrm{Au} / \mathrm{Ni} / \mathrm{Au}$ наносилась методом термического напыления (суммарная толщина $0.32 \mu \mathrm{m}$ ) и затем вжигалась для формирования омических контактов к структурам.

Для исследования спектральных свойств и мощности импульсного ТГц излучения ФПА монтировались на специальный юстируемый держатель производства „TERAVIL“ (см. рис. 2, $b$ ) и устанавливались в качестве источника в стандартную установку импульсной ТГц спектроскопии (ИТС) (рис. 2,a) [20]. В состав держателя входила юстируемая гиперполусферическая линза из высокоомного кремния диаметром $15 \mathrm{~mm}$, прижимаемая пружиной к обратной стороне подложки ФПА. Она позволяла уменьшить расходимость ТГц излучения из ФПА и повысить эффективность вывода излучения из подложки. Оптическое фемтосекундное излучение от лазерной системы Spectra Physics Tsunami $(\lambda=800 \mathrm{~nm}$, $\tau=80 \mathrm{fs}, f_{\mathrm{imp}}=80 \mathrm{MHz}$ ) разделялось на две части, одна из которых попадала на исследуемую ФПА, проходя через аттенюатор и микрообъектив, а вторая после прохождения линии задержки - на ФПА-детектор. Диапазоны изменения оптической мощности излучения и питающего напряжения на исследуемых ФПА составляли 0-75 mW и 0-30 V, соответственно. Большинство измерений проводилось при оптической мощности $19 \mathrm{~mW}$ и напряжении смещения $15 \mathrm{~V}$ во избежание повреждения ФПА.

При измерении спектральных свойств источника методом ИТС изначально регистрируется временной профиль электрического поля ТГц импульса посредством измерения напряжения на детектирующей ФПА в зависимости от задержки между оптическими импульсами на антеннах [21]. Данную зависимость затем можно пересчитать в спектр сигнала при помощи преобразования Фурье. В то же время необходимость юстировки ИТС спектрометра по множеству степеней свободы усложняет измерение абсолютной величины сигнала и ее сравнение для разных ФПА. Поэтому в данной работе применялся также калиброванный оптоакустический детектор ТГц излучения - ячейка Голея (GC-1P, Tydex), регистрирующий среднюю мощность ТГц сигнала ФПА. Этот детектор менее чувствителен к точности фокусировки ТГц излучения и к диаграмме направленности ТГц источника по сравнению с ФПА. При этом данный детектор не обладает спектральной чувствительностью и измеряет интегральный по всем частотам ТГц сигнал.

\section{Экспериментальные результаты}

Двумерное удельное сопротивление всех исследованных структур составляло более $10^{7} \Omega$. Это указывает на низкую концентрацию свободных носителей заряда в структурах на подложках (100) и (111)A, несмотря на легирование атомами $\mathrm{Si}$. По-видимому, большинство носителей заряда, созданных донорными или акцепторными атомами $\mathrm{Si}$ в высокотемпературных слоях $\mathrm{GaAs}: \mathrm{Si}$, были захвачены дефектами в соседних слоях LT-GaAs. Темновое сопротивление между электродами всех ФПА составляло более $100 \mathrm{M} \Omega$.

Характеристики генерируемого ФПА ТГц излучения для всех структур исследовались при идентичных условиях. Излучение фемтосекундного лазера мощностью $19 \mathrm{~mW}$ направлялось в зазор ФПА, к которой было приложено смещение $15 \mathrm{~V}$. На рис. 3 приведен пример временной формы и частотного спектра ТГц импульсов от дипольных антенн на структурах № 443-б/о и № 443-отж с ориентацией (111)А. Основные отличия в спектрах ТГц сигналов для разных структур состояли в положении максимума спектров в диапазоне от 0.1 до $0.5 \mathrm{THz}$ (см. табл. 1) и амплитуде сигналов.

В табл. 1 приведено сравнение характеристик ТГц излучения дипольных ФПА, изготовленных на различных структурах. Для нескольких образцов измерялась

Таблица 1. Параметры ТГц импульсов, генерируемых дипольными ФПА с шириной фотопроводящего зазора $20 \mu \mathrm{m}$

\begin{tabular}{c|c|c|c|c}
\hline Образец & $\begin{array}{c}\text { Ориентация } \\
\text { подложки }\end{array}$ & Отжиг & $\begin{array}{c}\text { Интегральная } \\
\text { мощность, nW }\end{array}$ & $\begin{array}{c}\text { Максимум } \\
\text { спектра, ТНz }\end{array}$ \\
\hline \multirow{2}{*}{ № 7 } & $(100)$ & б/о & 88 & 0.15 \\
\cline { 2 - 5 } & $(111) \mathrm{A}$ & б/о & 110 & 0.14 \\
\hline \multirow{2}{*}{ № 26 } & $(100)$ & отж & $0.2^{*}$ & 0.35 \\
\cline { 2 - 5 } & $(111) \mathrm{A}$ & отж & $56^{*}$ & 0.13 \\
\hline \multirow{2}{*}{ № 443 } & $(111) \mathrm{A}$ & б/о & $1.9^{*}$ & 0.47 \\
\cline { 2 - 5 } & $(111) \mathrm{A}$ & отж & 750 & 0.19
\end{tabular}

Примечание. * Значение рассчитано по данным ИТС. 

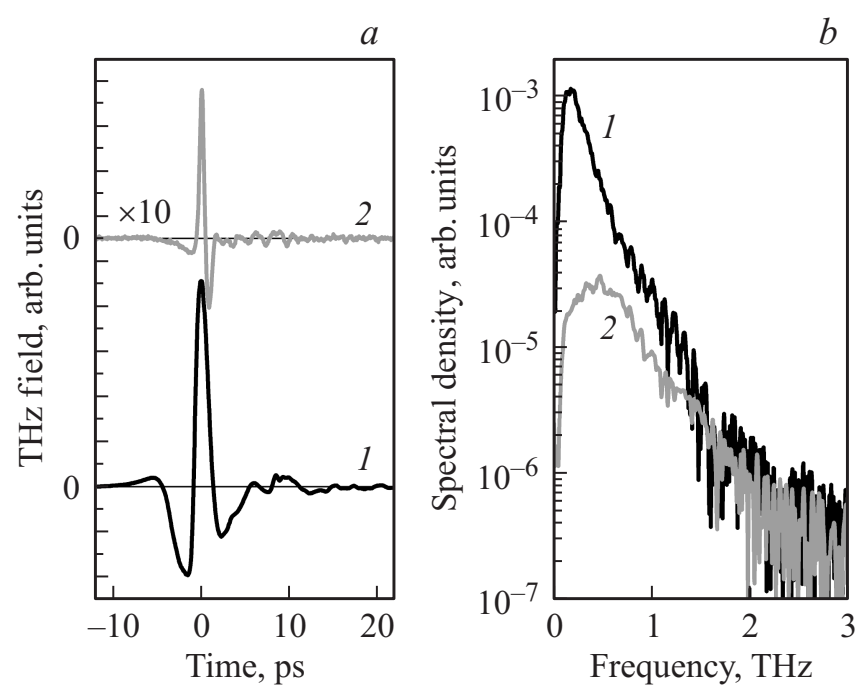

Рис. 3. Временная форма $(a)$ и спектральный состав $(b)$ ТГц излучения после (1) и до (2) отжига структуры № 443 (111)А.

интегральная по спектру мощность ТГц импульсов. Для образцов, мощность излучения которых не измерялась ячейкой Голея непосредственно, приведен пересчет интегральной мощности по данным ИТС. Для этого спектр ФПА интегрировался в диапазоне $0.01-1.5 \mathrm{THz}$ и нормировался на значение для ФПА 443отж.

Все ФПА, описанные в табл. 1, имеют одинаковую топологию электродов, но различаются условиями получения или ориентацией подложки структур \{LT$\mathrm{GaAs} / \mathrm{GaAs}: \mathrm{Si}\}$. Из данных измерений для обр. № 7 и № 26 следует, что ФПА, изготовленные на структурах с ориентацией (111)А, испускают более интенсивные импульсы ТГц излучения, чем аналогичные им на подложках (100). Отметим влияние высокотемпературного отжига структур на свойства соответствующих ФПА. Для структур № 7 и № 26, полученных при усредненном соотношении потоков $\mathrm{As}_{4}$ и $\mathrm{Ga} \gamma=25$, высокотемпературный отжиг привел к уменьшению мощности ТГц импульсов. Отжиг образца № 443, напротив, привел к увеличению мощности ФПА на несколько порядков. Отжиг структур на основе LT-GaAs приводит к изменению концентрации различных точечных дефектов, диффузии дефектов, объединению избыточных атомов As в кристаллические преципитаты. Поэтому различную зависимость мощности ТГц излучения ФПА № 443 и № 26, № 7 от отжига мы связываем с различным содержанием избыточных атомов мышьяка в LT-GaAs слоях соответствующих структур.

Наиболее интенсивные ТГц импульсы испускали ФПА на основе образца (443)-отж. При получении этой структуры как температура подложки, так и давление мышьяка различались для слоев LT-GaAs и GaAs: $\mathrm{Si}$. Коэффициент преобразования энергии оптических импульсов в ТГц для дипольной ФПА 443-отж. составлял порядка $3 \cdot 10^{-5}$ при работе с напряжением смещения $U_{\text {bias }}=15 \mathrm{~V}$ и достигал $1.2 \cdot 10^{-4}$ при $U_{\text {bias }}=30 \mathrm{~V}$. Для
ФПА в форме галстука-бабочки на этой же структуре при $U_{\text {bias }}=15 \mathrm{~V}$ эффективность оптико-ТГц конверсии составляла $2.5 \cdot 10^{-4}$, а средняя мощность ТГц излучения составляла $4.7 \mu \mathrm{W}$. Длительность импульсов ТГц излучения была существенно больше, чем от дипольных ФПА. Поэтому основная доля энергии ТГц импульсов от ФПА галстук-бабочка была сосредоточена в низкочастотной области $<0.3 \mathrm{THz}$ в соответствии с результатами работы [22].

Измерения интегральной мощности $W_{\mathrm{THz}}$ излучения дипольной антенны 443-отж в зависимости от приложенного напряжения и от мощности фемтосекундных импульсов приведены на рис. 4. Можно отметить, что в исследованном диапазоне напряжений $U_{\text {bias }}=5-30 \mathrm{~V}$ мощность ТГц излучения суперлинейно зависела от $U_{\text {bias }}[23,24]$. Экспериментальные зависимости $W_{\mathrm{THz}}\left(U_{\text {bias }}\right)$ довольно хорошо интерполировались степенной функцией вида $W_{\mathrm{THz}} \sim U_{\text {bias }}^{h}$, где $b=$ const. Значение показателя степени составило $b=1.6$ при мощности накачки $7.5 \mathrm{~mW}$ и $b=1.8$ при мощности накачки $19 \mathrm{~mW}$. Зависимость $W_{\mathrm{THz}}$ от мощности оптических импульсов накачки, приведенная на рис. 4 , является сублинейной и имеет вид кривой насыщения. Такую зависимость можно связать с экранированием приложенного электрического поля между электродами ФПА за счет возрастающей концентрации фотовозбужденных носителей заряда [22].

\section{Практическое применение}

Существуют приложения, для которых необходимо использовать низкочастотные ФПА, например ИТС растворов. С помощью дипольной ФПА № 443-отж в качестве ТГц генератора удалось расширить динамический диапазон импульсного ТГц спектрометра до $0.05 \mathrm{THz}$. Главным ТГц-маркером во всех растворах является в большей степени вода, так как она имеет сильное поглощение в этом диапазоне. Подавляющая часть био-
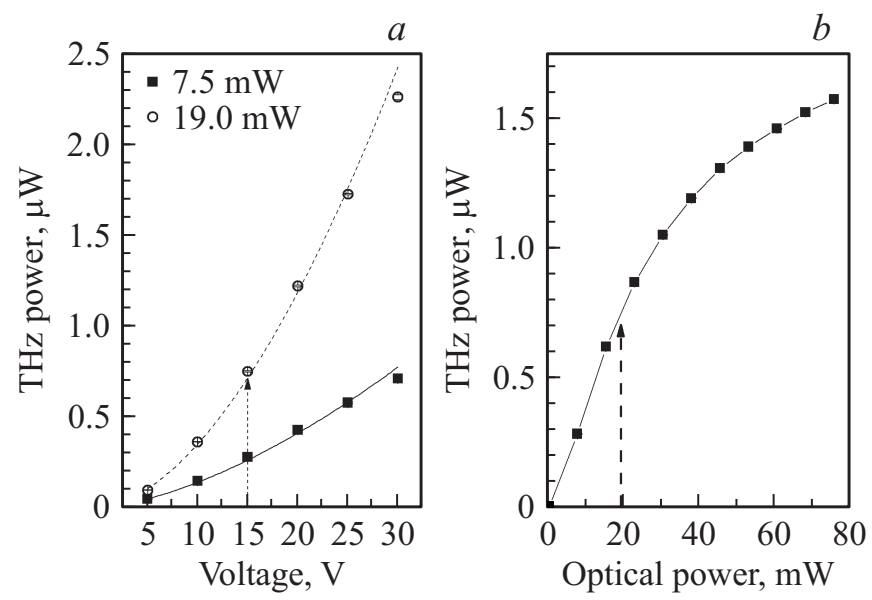

Рис. 4. Зависимости интегральной мощности излучения дипольной антенны 443-отж: $(a)$ от напряжения смещения для двух уровней оптической накачки $(7.5$ и $19 \mathrm{~mW}),(b)$ от мощности оптической накачки при напряжении смещения $15 \mathrm{~V}$. 

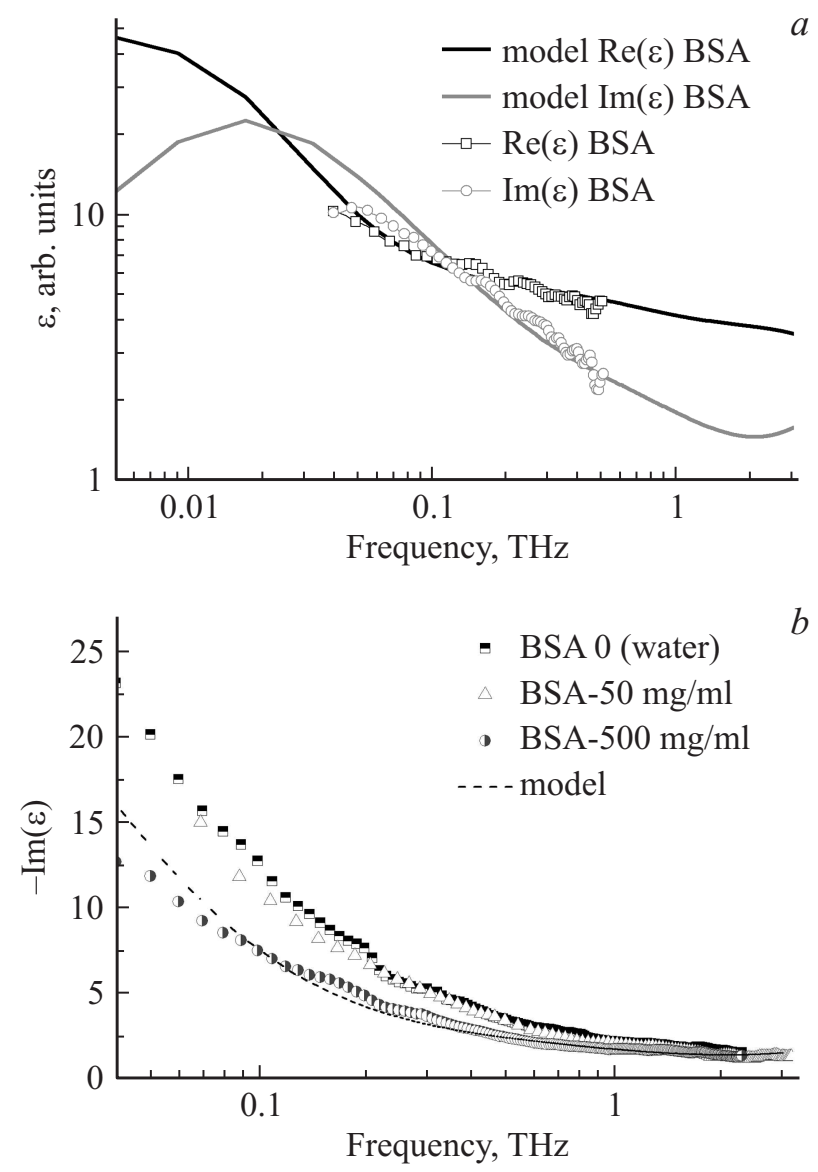

Рис. 5. Спектры $(a)$ действительной и мнимой частей диэлектрической функции $\varepsilon(f)$ водного раствора БСА с концентрацией $500 \mathrm{mg} / \mathrm{ml}$ при $T=22^{\circ} \mathrm{C}$. Точки — экспериментальные данные, сплошные кривые - модели Дебая, $(b)$ мнимой части диэлектрической функции $\varepsilon(f)$ водного раствора БСА с концентрацией $0 \mathrm{mg} / \mathrm{ml}$ - вода (квадраты), $50 \mathrm{mg} / \mathrm{ml}$ (треугольники), $500 \mathrm{mg} / \mathrm{ml}$ (круглые точки), штриховая кривая модель Дебая при $T=22^{\circ} \mathrm{C}$.

молекул, в том числе белки, находятся в организме в растворенном в воде состоянии. Известно, что измеряемая с помощью ТГц спектроскопии характеристика растворов - это состояние воды, преобладающей в биологических образцах (растворах и тканях) [25]. Вода может находиться в свободном и связанном состояниях; каждое из них вносит собственный вклад в амплитуду ТГц спектров пропускания/отражения [26]. Изменения пропорций свободной и связанной воды, изменение времен релаксации для каждого из этих состояний воды - все это проявляется в ТГц диапазоне частот [27]. Стандартные ТГц импульсные спектрометры обычно не захватывают центр низкочастотного „пика“ поглощения воды (в области $0.02 \mathrm{THz}$ ), а только его высокочастотный „хвоск“, начиная с частоты $0.1 \mathrm{THz}$. Поэтому для достоверного измерения малых концентраций вещества в водных растворах необходимо расширение спектрального диапазона в низкие частоты, вплоть до $0.02 \mathrm{THz}$. Использование дипольной ФПА № 443-отж позволило просветить водный образец толщиной $0.5 \mathrm{~mm}$ излучением с частотами вплоть до $1 \mathrm{THz}$. Для стабильного измерения низких частот $(\sim 0.05 \mathrm{THz})$ применялась длинная временная выборка - от -20 до 40 ps относительно центра ТГц импульса. Проверка эффективности дипольной ФПА № 443-отж была выполнена при регистрации ТГц спектров поглощения раствора бычьего сывороточного альбумина (БСА) (Sigma, США) - основного белка, входящего в состав сыворотки крови, в воде с концентрацией 50 и $500 \mathrm{mg} / \mathrm{ml}$. Методика эксперимента и обработки результатов приведены в наших предыдущих работах [28-30].

Наиболее простым и точным выражением, описывающим диэлектрическую функцию $\varepsilon(\omega)$ водных растворов в диапазоне частот $0.05-5 \mathrm{THz}$, является известная двухкомпонентная релаксационная модель Дебая [26,30,31]. Также известно, что самая информативная область для растворов находится в районе пика дебаевской „медленной релаксации“, которая отвечает за переориентацию водородных связей молекул воды и находится в районе $20 \mathrm{GHz}$. В связи с этим, для ИТС важно иметь излучатель, который позволяет изучать наиболее информативные спектры в низкочастотном ТГц диапазоне $(0.05-0.4 \mathrm{THz})$.

Чтобы описать спектры диэлектрической функции $\varepsilon(\omega)$ раствора белка, мы аппроксимируем экспериментальные комплексные спектры к модели Дебая (1) с изменением только амплитуды первого члена „медленной релаксации“" [26,32]:

$$
\begin{aligned}
\varepsilon(\omega, C)= & \varepsilon_{\infty}+\frac{\Delta \varepsilon_{1}\left(1-C \cdot 1.3 \cdot 10^{-3}\right.}{1+i \omega \tau_{1}} \\
& +\frac{\Delta \varepsilon_{2}}{1+i \omega \tau_{2}}+\frac{A_{1}}{\omega_{01}^{2}-\omega^{2}+i \gamma_{01} \omega},
\end{aligned}
$$

где $\tau_{1}$ - время процесса „медленной“ релаксации, $\tau_{2}$ время процесса „быстрой“ релаксации, $\Delta \varepsilon_{1}$ - вклад в диэлектрическую проницаемость первого члена, $\Delta \varepsilon_{2}-$ вклад в диэлектрическую проницаемость второго члена, $A_{1}$ - амплитуда, $\omega_{01}=2 \pi f-$ циклическая частота. Сумма всех амплитуд должна быть равна известной статической диэлектрической проницаемости $\varepsilon_{s}(2)$ (для воды при температуре $\left.25^{\circ} \mathrm{C} \varepsilon_{s}=78.5\right)$ :

$$
\varepsilon_{s}=\varepsilon_{\infty}+\Delta \varepsilon_{1}+\frac{A_{1}}{\omega_{01}^{2}}+\text { ldots } .
$$

В основе модели Дебая (1) лежат следующие физические процессы: $\tau_{1}$ - общее время переориентации объемных молекул воды с водородной связью (разрыв и создание новых водородных связей), $\tau_{2}$ - быстрая релаксация при ионном процессе, которая происходит без взаимодействия водородных связей, то есть релаксация свободной воды. Преобразование части объема молекул воды из свободного в связанное состояние описывается для ТГц диапазона как величина амплитуды медленной релаксации. В табл. 2 представлены параметры модели для спектра воды. 
Таблица 2. Используемые параметры модели ТГц спектра воды

\begin{tabular}{c|c|c|c|c|c|c|c|c|c|c}
\hline$f, \mathrm{THz}$ & $\varepsilon_{s}$ & $\varepsilon_{\infty}$ & $\Delta \varepsilon_{1}$ & $\tau_{1}, \mathrm{ps}$ & $\Delta \varepsilon_{2}$ & $\tau_{1} \mathrm{fs}$ & $A_{1} / \omega_{01}^{2}$ & $\omega_{01}, \mathrm{THz}$ & $\gamma_{01}, \mathrm{THz}$ & $T,{ }^{\circ} \mathrm{C}$ \\
\hline $0.1-2.7$ & 78.5 & $2.5 \pm 0.1$ & 75.6 & $9.5 \pm 0.8$ & $1.47 \pm 0.05$ & $230 \pm 30$ & 1.14 & 5.3 & 5.35 & 22
\end{tabular}

При низкой частоте (ниже 0.1 ТГц) вклад медленного релаксационного члена в общее поглощение составляет 98\% [27]. Таким образом, на низких частотах (0.1 ТГц) преобладает медленная релаксация $\Delta \varepsilon_{1}, \tau_{1}$. Для прогнозирования изменения ТГц спектра $\varepsilon(\omega)$ раствора при увеличении концентрации белка (БСА) нужно умножить $\Delta \varepsilon_{1}$ на величину $1-C(\mathrm{mg} / \mathrm{ml}) \cdot 1.3 \cdot 10^{-3}$.

При аппроксимации экспериментального спектра раствора БСА с концентрацией $500 \mathrm{mg} / \mathrm{ml}$ модельными данными, амплитуда первого члена модели Дебая составила $\Delta \varepsilon_{1}=46 \pm 3$. Таким образом, экспериментально полученная диэлектрическая проницаемость раствора БСА совпала с известной $[26,30,31]$ моделью.

Полученные нами спектры действительной и мнимой частей диэлектрической проницаемости водного раствора БСА представлены на рис. 5,a. В данном случае использовалась нетипично большая концентрация БСА $(500 \mathrm{mg} / \mathrm{ml})$ по сравнению с концентрацией альбумина в сыворотке крови человека $(74 \mathrm{mg} / \mathrm{ml})$, для того, чтобы сильнее проявилось изменение отклика связанной воды.

Спектры мнимой части диэлектрической проницаемости для водного раствора БСА разной концентрации приведены на рис. 5, $b$. Видно, что наибольшие отличия в спектрах наблюдаются в низких частотах. Способность отличать растворы БСА с концентрацией $50 \mathrm{mg} / \mathrm{ml}$ от воды является актуальной задачей, для выполнения которой необходимы источник и приемник с хорошим динамическим диапазоном, особенно с высокой разрешающей способностью в низких частотах. Видно, что различия между разными концентрациями раствора БСА наиболее выражены при частотах ниже $0.2 \mathrm{THz}$.

Несмотря на то, что область между СВЧ и ТГц-ТДС $(20-70 \mathrm{GHz})$ является технически труднодоступной, нам удалось получить достоверные, актуальные данные по раствору БСА. Благодаря разработанному низкочастотному генератору повышается информативность изучения биологических сред, так как основной их составляющей является вода. При изменении концентрации раствора, вода переходит из свободного состояния в связанное и обратно, что хорошо наблюдается в диапазоне $20 \mathrm{GHz}$, который соответствует пику „медленной“ дебаевской релаксации.

\section{Обсуждение}

В работе исследованы новые структуры \{LT$\mathrm{GaAs} / \mathrm{GaAs}: \mathrm{Si}\}$ в качестве материала для ТГц ФПА. Показано, что при использовании подложек с ориентацией (111)А и при выборе подходящих условий эпитаксиального роста слоев LT-GaAs и GaAs: Si получаемые структуры являются подходящими для эффективных ФПА-источников ТГц волн. ФПА, изготовленные на структуре № 443, испускают мощные ТГц импульсы, сравнимые по амплитуде с коммерческими ФПА. Также ФПА на структуре № 443-отж обладают самой высокой выходной мощностью среди всех LT-GaAs ФПА с аналогичной топологией, изготовленных авторами работы. Влияние ориентации подложки на свойства структур \{LT-GaAs/GaAs:Si\} мы связываем с двумя факторами. Во-первых, ориентация подложки влияет на тип легирующих атомов $\mathrm{Si}$, которые встраиваются в решетку GaAs при росте. Так, на подложках GaAs (100) Si преимущественно проявляет донорные свойства, а на подложках (111)А при низких значениях $\gamma$ - акцепторные. Акцепторы и доноры соответственно увеличивают и уменьшают в слоях LT-GaAs концентрацию заряженных антиструктурных дефектов $\mathrm{AsGa}$, являющихся основными центрами безызлучательной рекомбинации. Во-вторых, ориентация подложки при росте влияет на концентрацию и тип образующихся точечных дефектов и на другие свойства слоев LT-GaAs. Именно совокупность физических свойств слоев LT-GaAs, таких как подвижность носителей заряда, время жизни, сопротивление, поле пробоя существенно определяет характеристики ФПА. Однако исследований свойств эпитаксиальных пленок GaAs (111)A, полученных в низкотемпературном режиме, в литературе практически нет. В работе [18] показано, что структуры $\{\mathrm{LT}-\mathrm{GaAs} / \mathrm{GaAs}: \mathrm{Si}\}$ (111)A являются монокристаллическими до некоторой толщины порядка 300 nm, выше которой становятся поликристаллическими. После отжига структур в поликристаллических областях не наблюдались преципитаты As, в отличие от структур на подложках (100). Поликристалличность структур \{LT-GaAs/GaAs: Si $\}$ (111)A изменяет механизмы диффузии и рекомбинации дефектов в LT-GaAs при отжиге, а также существенно изменяет характер движения неравновесных носителей заряда в зазоре ФПА, приводя к рассеянию. Результаты работы тем более удивительны, поскольку оптимальными структурами LT-GaAs для ФПА-источников ТГц излучения считаются монокристаллические структуры с высокой подвижностью электронов.

\section{Заключение}

В работе предложен материал на основе низкотемпературного GaAs для получения терагерцовых фотопроводящих антенн. Структуры содержат активные слои из LT-GaAs вместе с вспомогательными легирующими акцепторными слоями на основе GaAs:Si. Структуры 
были получены методом МЛЭ на подложках (111)А при оптимизированных условиях роста для слоев LT-GaAs и $\mathrm{GaAs}: \mathrm{Si}$. При мощности оптической накачки $19 \mathrm{~mW}$ и напряжении смещения $30 \mathrm{~V}$ фотопроводящая антенна на оптимизированной структуре $\{\mathrm{LT}-\mathrm{GaAs} / \mathrm{GaAs}: \mathrm{Si}\}$ (111)A испускала ТГц импульсы со средней мощностью $2.3 \mu \mathrm{W}$ при частоте следования $80 \mathrm{MHz}$, эффективность преобразования составила $1.2 \cdot 10^{-4}$. Было продемонстрировано, что изменением топологии ФПА можно существенно увеличить эффективность оптико-ТГц преобразования. Показано, что зависимость интегральной мощности $\mathrm{THz}$ импульсов ФПА на основе \{LT-GaAs/GaAs: $\mathrm{Si}\}$ (111)A структуры от приложенного напряжения является суперлинейной, а от мощности оптической накачки имеет вид кривой насыщения.

Частотный спектр исследованных ФПА сосредоточен в области низких частот. Максимумы спектров ФПА располагались при $0.1-0.5 \mathrm{THz}$, а спектральная плотность мощности сосредоточена на частотах менее $1 \mathrm{THz}$. Низкочастотные ФПА большой мощности перспективны для измерения водных растворов белков, что может найти применение в биологии и медицине. С использованием изготовленных ФПА были получены достоверные и актуальные данные по раствору белка в труднодоступном диапазоне частот $20-70 \mathrm{GHz}$.

\section{Благодарности}

Авторы благодарны Назарову М.М. и Черкасовой О.П. за ценные советы и помощь по интерпретации результатов терагерцовой спектроскопии растворов.

\section{Финансирование работы}

Работа выполнена при финансовой поддержке РФФИ в части изготовления эпитаксиальных структур и ФПА (грант № 18-32-20207 мол-а-вед) и спектроскопии водных растворов белков (грант № 17-00-00275 (17-0000270) и № 19-52-55004 Китай_a), а также при поддержке Министерства науки и высшего образования в рамках выполнения работ по Государственному заданию ФНИЦ „Кристаллография и фотоника“ РАН в части исследования процесса преобразования ФПА лазерного излучения ближнего ИК диапазона в терагерцовое излучение.

\section{Конфликт интересов}

Авторы заявляют, что у них нет конфликта интересов.

\section{Список литературы}

[1] Krotkus A. // J. Phys. D: Appl. Phys. 2010. V. 43. P. 273001. doi 10.1088/0022-3727/43/27/273001

[2] Burford N.M., El-Shenawee M.O. // Opt. Eng. 2017. V. 56. N 1. P. 010901. doi 10.1117/1.OE.56.1.010901

[3] Lepeshov S., Gorodetsky A., Krasnok A., Rafailov E., Belov P. // Laser \& Photonics Reviews. 2017. V. 11. N 1. P. 1600199. doi 10.1002/1por.201600199
[4] Yachmenev A.E., Lavrukhin D.V., Glinskiy I.A., Zenchenko N.V., Goncharov Y.G., Spektor I.E., Khabibullin R.A., Otsuji T., Ponomarev D.S. // Optical Engineering. 2019. V. 59(6). P. 061608. doi 10.1117/1.OE.59.6.061608

[5] Yardimci N.T., Jarrahi M. // Small. 2018. V. 14. N 44. P. 1802437. doi 10.1016/0039-6028(95)00750-4

[6] Yang S.H., Hashemi M.R., Berry C.W., Jarrahi M. // IEEE Trans. on Terahertz Science and Tech. 2014. V. 4(5). P. 575-581. doi 10.1109/TTHZ.2014.2342505

[7] Krotkus A., Coutaz J.L. // Semiconductor science and technology. 2005. V. 20. N 7. P. S142. doi 10.1088/0268$1242 / 20 / 7 / 004$

[8] Lavrent'eva L.G., Vilisova M.D., Preobrazhenskii V.V., Chaldyshev V.V. // Russian Physics Journal. 2002. V. 45. N 8. P. 735. doi 10.1023/A:1021965211576

[9] Stellmacher M., Nagle J., Lampin J.F., Santoro P., Vaneecloo J., Alexandrou A. // J. Appl. Phys. 2000. V. 88. N 10. P. 6026. doi 10.1063/1.1285829

[10] Gregory I.S., Baker C., Tribe W.R., Evans M.J., Beere H.E., Linfield E.H., Missous M. // Appl. Phys. Lett. 2003. V. 83. N 20. P. 4199. doi 10.1063/1.1628389

[11] Moon K., Choi J., Shin J.H., Han S.P., Ko H., Kim N., Park K.H. // ETRI Journal. 2014. V. 36. N 1. P. 159. doi 10.4218/etrij.14.0213.0319

[12] Krotkus A., Bertulis K., Dapkus L., Olin U., Marcinkevicius S. // Appl. Phys. Lett. 1999. V. 75. N 21. P. 3336. doi 10.1063/1.125343

[13] Eusebe H., Roux J.F., Coutaz J.L., Krotkus A. // J. Appl. Phys. 2005. V. 98. N 3. P. 033711. doi 10.1063/1.2001151

[14] Liu X., Prasad A., Chen W.M., Kurpiewski A., Stoschek A., Liliental-Weber Z., Weber E.R. // Appl. Phys. Lett. 1994. V. 65. N 23. P. 3002-3004. doi 10.1063/1.112490

[15] Галиев Г.Б., Климов Е.А., Клочков А.Н., Копылов В.Б., Пушкарев С.С. // ФТП. 2019. Т. 53. № 2. С. 258; Galiev G.B., Klimov E.A., Klochkov A.N., Kopylov V.B., Pushkarev S.S. // Semicond. 2019. V. 53. N 2. P. 246. doi 10.1134/S1063782619020088

[16] Kuznetsov K.A., Galiev G.B., Kitaeva G.Kh., Kornienko V.V., Klimov E.A., Klochkov A.N., Leontyev A.A., Pushkarev S.S., Maltsev P.P. // Laser Phys. Lett. 2018. V. 15. P. 076201. doi 10.1088/1612-202X/aac7bb

[17] Галиев Г.Б., Грехов М.М., Китаева Г.Х., Климов Е.А., Клочков А.Н., Коленцова О.С., Корниенко В.В., Кузнецов К.А., Мальцев П.П., Пушкарев С.С. // ФТП. 2017. Т. 51. № 3. C. 322; Galiev G.B., Grekhov M.M., Kitaeva G.Kh., Klimov E.A., Klochkov A.N., Kolentsova O.S., Kornienko V.V., Kuznetsov K.A., Maltsev P.P., Pushkarev S.S. // Semicond. 2017. V. 51. N 3. P. 310. doi 10.1134/S1063782617030071

[18] Галиев Г.Б., Трунькин И.Н., Васильев А.Л., Васильевский И.С., Виниченко А.Н., Климов Е.А., Клочков А.Н., Мальцев П.П., Пушкарев С.С. // Кристаллография. 2019. T. 64. N 2. C. 184; Galiev G.B., Trunkin I.N., Vasiliev A.L., Vasil'evskii I.S., Vinichenko A.N., Klimov E.A., Klochkov A.N., Maltsev P.P., Pushkarev S.S. // Crystallogr. Reports. 2019. V. 64. P. 205.

[19] Галиев Г.Б., Климов Е.А., Клочков А.Н., Пушкарев С.С., Мальцев П.П. // ФТП. 2018. Т. 52. № 3. С. 395; Galiev G.B., Klimov E.A., Klochkov A.N., Pushkarev S.S., Maltsev P.P. // Semiconductors. 2018. V. 52. N 3. P. 376. doi $10.1134 / \mathrm{S} 1063782618030119$ 
[20] Nazarov M.M., Shkurinov A.P., Kuleshov E. A., Tuchin V.V.// Quantum electronics. 2008. V. 38. N 7. P. 647. doi 10.1070/QE2008v038n07ABEH013851

[21] Zhang X.C., Xu J. Introduction to THz wave photonics. New York : Springer, 2010. V. 29. 246 p.

[22] Tani M., Matsuura S., Sakai K., Nakashima S.I. // Appl. Optics. 1997. V. 36. N 30. P. 7853. doi 10.1364/AO.36.007853

[23] Zhang J., Hong Y., Braunstein S.L., Shore K.A. // IEE Proceedings-Optoelectronics. 2004. V. 151. N 2. P. 98. doi 10.1049/ip-opt:20040113.

[24] Jepsen P.U., Jacobsen R.H., Keiding S.R. // JOSA B. 1996. V. 13. N 11. P. 2424. doi 10.1364/JOSAB.13.002424

[25] Nazarov M.M., Cherkasova O.P., Shkurinov A.P. // Quantum Electronics. 2016. V. 46. N 6. P. 488. doi 10.1070/QEL16107

[26] Smolyanskaya O.A, Chernomyrdin N.V., Konovko A.A., Zaytsev K.I., Ozheredov I.A., Cherkasova O.P., Nazarov M.M., Guillet J.P., Kozlov S.A., Kistenev Yu.V., Coutaz J.-L., Mounaix P., Vaks V.L., Son J.-H., Cheon H., Wallace V.P., Feldman Yu., Popov I., Yaroslavsky A.N., Shkurinov A.P., Tuchin V.V. // Progress in Quantum Electronics. 2018. V. 62. P. 1. doi 10.1016/j.pquantelec.2018.10.001

[27] Shiraga K., Adachi A., Nakamura M., Tajima T., Ajito K., Ogawa Y. // J. Chem. Phys. 2017. V. 146. N 10. P. 105102. doi $10.1063 / 1.4978232$

[28] Nazarov M., Shkurinov A., Tuchin V.V., Zhang X.C. // Handbook of photonics for biomedical science / Ed. by V.V. Tuchin, CRC Press, 2010, P. 591.

[29] Angeluts A.A., Balakin A.V., Evdokimov M.G., Esaulkov M.N., Nazarov M.M., Ozheredov I.A., Sapozhnikov D.A., Solyankin P.M., Cherkasova O.P., Shkurinov A.P. // Quantum Electronics. 2014. V. 44. N 7. P. 614.

[30] Cherkasova O.P., Nazarov M.M., Angeluts A.A., Shkurinov A.P. // Optics and Spectroscopy. 2016. V. 120. N 1. P. 50.

[31] Raicu V., Feldman Y. Dielectric relaxation in biological systems: Physical principles, methods, and applications. New York: Oxford University Press, 2015.

[32] Nazarov M.M., Cherkasova O.P., Shkurinov A.P. // J. Infrared Millimeter and Terahertz Waves. 2018. V. 39. N 9. P. 840-853. doi $10.1007 / \mathrm{s} 10762-018-0513-3$ 